\title{
Numerical analysis of multiphase flow through axial vortex tube cyclone separators
}

\author{
Gopalakrishnan $\mathrm{B}^{1}$ Arul Prakash $\mathrm{K}^{1}$ \\ ${ }^{1}$ Department of Applied Mechanics, IIT Madras, Chennai, India
}

\begin{abstract}
Axial cyclone separators are used as primary filtration components in many internal combustion engine applications. A numerical study of gas-solid flow through an axial cyclone separator is carried out by Eulerian-Lagrangian CFD approach, which uses discrete phase modelling (DPM) of particles and solution of incompressible turbulent Navier-Stokes equations with RNG $k-\varepsilon$ closure model. The effective filtration of inlet air from dust particles is an important requirement for prolonged engine life cycle. In axial cyclone separators, a helical swirl generator is used for imparting swirling motion to the particle laden flow and particles are filtered by centrifugal separation and then scavenged using a vortex tube. In the present work, the modelling done in OpenFOAM successfully captures the features of the swirling flow, along with particle-gas and particle-wall interactions for a varying size distribution at the inlet. Studies were aimed at analysing the effect of particles and its effect on improving the performance of the filter.
\end{abstract}

Keywords: Axial cyclone separator, Filtration efficiency, Helical swirl generator, OpenFOAM, Discrete phase modelling

\section{NOMENCLATURE}

$\rho$ Density of the fluid

$\rho_{p}$ Density of the particle

$\mu$ Dynamics viscosity of the fluid

$\mu_{\mathrm{t}}$ Eddy viscosity

$\mathrm{C}_{\mathrm{p}}$ Specific heat capacity

$\mathrm{k}$ Turbulent kinetic energy

$\varepsilon$ Rate of dissipation of turbulence kinetic energy

$\mathrm{u}_{\mathrm{p}}$ Particle velocity

$\mathrm{u}$ Fluid velocity

\section{Introduction}

Cyclone separators are devices which are used for separation of denser particles from a carrying fluid. They have applications in various industries such as processing industries, petrochemical industry and automobile industry for filtering off dust particles from the gas flow. Their wide use is mainly due to its low cost, simple design, low maintenance and adaptability. The main principle behind the working of a cyclone filter is centrifugal separation, where the density difference between the fluid and the gas is used to separate the denser particles from the lighter fluid.

Based on the mode of inlet, cyclone separators are classified into tangential and axial cyclones. The multiphase gas solid flow enters the cyclone, undergoes a swirling motion due to the helical swirl generator, and the particles which are heavier than air gets thrown into the periphery. The clean air is separated out with the help of a vortex tube. Although there is much research being done [1-2] on the tangential cyclones for studying its flow field and improving its performance, there have been limited research on the axial flow separators. Klusjo et al. [4] has done considerable research on axial cyclone with cone tip. The variation of an outlet tube with a tip blade was analysed. In his work he also experimented on the cone tip dimensions and flow rates. Ramachandran et al. [7] performed analytical studies on a general axial swirl tube separator. He developed analytical equations for pressure drop and efficiency based on fluid dynamic theory and with suitable assumptions.

The cyclone separators find their application in the effective filtration of inlet air in internal combustion engines. There has been very few research done on the improvement of performance of an axial cyclone separator. An improved filter performance results in prolonged engine life cycle in internal combustion engine. The major parameters of interest in a cyclone separator are the pressure drop across the filter and the particle filtration efficiency. The present study focuses on numerically simulating the flow field inside an axial vortex tube cyclone separator by CFD methods and tracking the trajectory of particles by discrete phase modelling techniques. The important parameters of interest in a cyclone separator are the pressure drop across the filter and the filtration efficiency. 
A preliminary study on the variation of different parameters and its effect on the output is performed. Studies are done to analyse the trajectory of particles, and how the size of the particles affect the filtration efficiency. Different behaviors of the particles are studied with respect to its implications on the filter performance.

\section{Analysis and modelling}

\subsection{Governing equations}

The flow field is obtained by numerically solving the incompressible Navier-Stokes equations given by:

$$
\nabla \cdot V=0
$$

$$
\begin{aligned}
& \frac{\partial \mathrm{u}}{\partial \mathrm{t}}+(\mathrm{V} \cdot \nabla) \mathrm{u}=-\frac{1}{\rho} \frac{\partial \mathrm{p}}{\partial \mathrm{x}}+\left(\mathrm{v}+\mathrm{v}_{\mathrm{t}}\right) \nabla^{2} \mathrm{u} \\
& \frac{\partial \mathrm{v}}{\partial \mathrm{t}}+(\mathrm{V} \cdot \nabla) \mathrm{v}=-\frac{1}{\rho} \frac{\partial \mathrm{p}}{\partial \mathrm{y}}+\left(v+v_{\mathrm{t}}\right) \nabla^{2} \mathrm{v} \\
& \frac{\partial \mathrm{w}}{\partial \mathrm{t}}+(\mathrm{V} \cdot \nabla) \mathrm{w}=-\frac{1}{\rho} \frac{\partial \mathrm{p}}{\partial \mathrm{z}}+\left(v+v_{\mathrm{t}}\right) \nabla^{2} \mathrm{w}
\end{aligned}
$$

The turbulent viscosity, $\mu_{\mathrm{t}}$ can be computed from the turbulent kinetic energy, $\mathrm{k}$, and its dissipation rate, given by:

$$
\begin{gathered}
\mu_{\mathrm{t}}=\mathrm{C}_{\mu} \rho \frac{\mathrm{k}^{2}}{\varepsilon} \\
\nu_{\mathrm{t}}=\frac{\mu_{\mathrm{t}}}{\rho}
\end{gathered}
$$

The particle trajectories are obtained by integrating the force balance equation on the particle, and can be written as:

$$
\frac{\mathrm{Du}_{\mathrm{p}}}{\mathrm{dt}}=\mathrm{F}_{\mathrm{d}}\left(\mathrm{u}-\mathrm{u}_{\mathrm{p}}\right)+\mathrm{g} \frac{\left(\rho_{p}-\rho\right)}{\rho_{p}}
$$

Where $F_{d}\left(u-u_{p}\right)$ is the drag force per unit particle mass, $\mathrm{u}$ represents fluid phase velocity, $\mathrm{u}_{\mathrm{p}}$ represents particle phase velocity.

$R_{e}$ is the relative Reynolds number given by

$$
R_{e}=\frac{\rho D_{p}\left(u-u_{p}\right)}{\mu}
$$

Where $\rho$ is the density of the particle, $D_{p}$ is the particle diameter and $\mu$ is the fluid dynamic viscosity.

$$
F_{d}=\left(\frac{18 \mu}{\rho_{p} D_{p}^{2}}\right)\left(\frac{R_{e} C_{D}}{24}\right)
$$

$\mathrm{C}_{\mathrm{d}}$ is known as the drag co efficient and is dependent on the drag law used. A spherical drag model is used and the value of $C_{d}$ is obtained from literature [3].

\subsection{Turbulence model}

The suitable selection of turbulence model is important. for accurately predicting the highly turbulent flow. The RNG model was developed using Re-Normalisation
Group (RNG) methods to renormalize the NavierStokes equation [8].

The transport equations for $\mathrm{k}$ and $\varepsilon$ are given by:

$$
\frac{\partial}{\partial \mathrm{t}}(\rho \mathrm{k})+\frac{\partial}{\partial \mathrm{x}_{\mathrm{i}}}\left(\rho \mathrm{ku}_{\mathrm{i}}\right)=\frac{\partial}{\partial \mathrm{x}_{\mathrm{j}}}\left[\left(\mu+\frac{\mu_{\mathrm{t}}}{\sigma_{\mathrm{k}}}\right) \frac{\partial \mathrm{k}}{\partial \mathrm{x}_{\mathrm{j}}}+\mathrm{P}_{\mathrm{k}}-\rho \varepsilon\right]
$$

$$
\frac{\partial}{\partial \mathrm{t}}(\rho \varepsilon)+\frac{\partial}{\partial \mathrm{x}_{\mathrm{i}}}=\frac{\partial}{\partial \mathrm{x}_{\mathrm{j}}}\left[\left(\mu+\frac{\mu_{\mathrm{t}}}{\sigma_{\varepsilon}}\right) \frac{\partial \varepsilon}{\partial \mathrm{x}_{\mathrm{j}}}+\mathrm{C}_{1 \varepsilon} \frac{\varepsilon}{\mathrm{k}} \mathrm{P}_{\mathrm{k}}-\mathrm{C}_{2 \varepsilon}^{*} \rho \frac{\varepsilon^{2}}{\mathrm{k}}\right]
$$

$$
\mathrm{C}_{2 \varepsilon}^{*}=\mathrm{C}_{2 \varepsilon}+\frac{\mathrm{C}_{\mu} \eta^{3}\left(1-\eta / \eta_{0}\right)}{1+\beta \eta^{3}}
$$

Where $\mathrm{P}_{\mathrm{k}}$ is the production term given by

$$
\begin{aligned}
& P_{k}=\mu_{t} S^{2} \\
& \eta=S \frac{k}{\varepsilon}
\end{aligned}
$$

$\mathrm{S}$ is the of mean rate of strain tensor defined as

$$
S=\left(2 S_{i j} S_{i j}\right)^{0.5}
$$

The constants used are similar to standard k- $\varepsilon$ model and are taken as $\mathrm{C} \mu=0.0845, \sigma_{\varepsilon}=0.7194, \sigma_{\mathrm{k}}=0.7194$, $\mathrm{C}_{\varepsilon 1}=1.42, \mathrm{C}_{\varepsilon 2}=1.68, \eta_{0}=4.38, \beta=0.012$.

\subsection{Computational domain and boundary conditions}

The modelling of blade geometry is done in SolidWorks and the computational domain is generated as shown in Figure 1. A fixed velocity of $13.36 \mathrm{~m} / \mathrm{s}$ is given at the inlet. The domain walls are given no slip boundary condition. At the outlet, zero gauge pressure is given as pressure boundary condition. The flow field is solved using a combination of SIMPLE and PISO algorithm (PIMPLE) and the Discrete Phase Modelling (DPM) of particles is done for calculating the trajectories using Lagrangian solvers in the opensource $\mathrm{C}++$ package OpenFOAM.

The three types of boundary conditions that can be provided for particles are escape, trap and rebound. The inlet and outlet faces are given escape boundary condition. The filter walls are given rebound boundary condition. The particles with a mass density of $2500 \mathrm{~kg} / \mathrm{m}^{3}$ are injected with the same velocity as that of the incoming flow with a mass concentration of $2 \mathrm{~g} / \mathrm{m}^{3}$. 


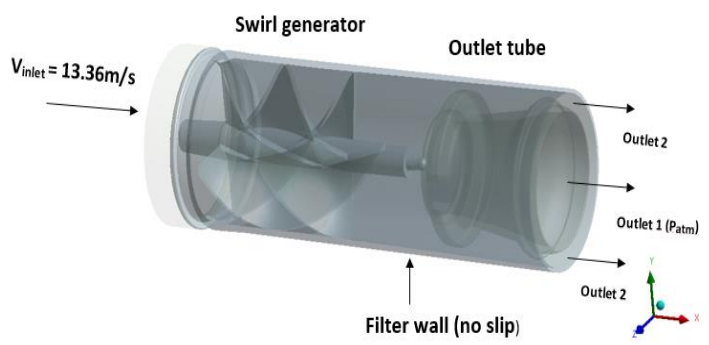

Figure 1. Computational domain

Initially, it is essential to check whether the particles affect the main flow field. This is done by enabling two way coupling between the flow and the particles. The discrete phase solver DPMFoam is used for solving the two way coupled equations with particle tracking. Extensive validation of DPMFoam is done by Fernandes et al. [11].

Since the particle concentration is very low, the flow parameters are found to be unaffected by the particles. Hence one way coupling have been found to be sufficient for further calculations. The equations are discretised to second order accuracy. The convergence criteria is given as $10^{-6}$ for velocity and $10^{-5}$ for pressure.

Table 1. Grid Independence test.

\begin{tabular}{|c|c|}
\hline $\begin{array}{c}\text { Number of } \\
\text { elements }\end{array}$ & $\begin{array}{c}\text { Pressure } \\
\text { drop(Pa) }\end{array}$ \\
\hline 1624447 & 954.2 \\
\hline 3045752 & 942.5 \\
\hline 4447587 & 945.7 \\
\hline
\end{tabular}

To obtain mesh independent solution, numerical analysis with different mesh sizes are carried out (Table 1). The pressure drop across the filter is compared for different cases. From the results the variation in pressure drop value is found to be less than $5 \%$ between 3 and 4 million mesh elements and hence a mesh size of about 3 million is chosen for simulations.

\section{Results and discussion}

Figure 2 shows the pressure contours across mid plane. Since the outlet pressure is gauge pressure, the pressure value at the inlet constitutes the pressure drop. Due to the presence of a protruding output shaft, there is a peak in the pressure value in the center. The face average of the pressure distribution is taken as the pressure drop.

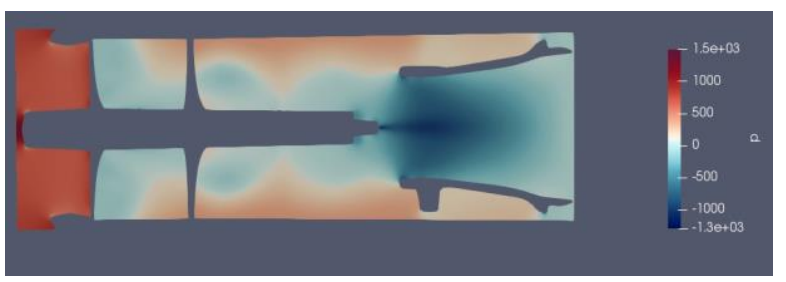

Figure 2. Pressure contour across mid-section
The region between the swirl generator and the vortex tube known as the separation region. Here, a negative pressure region is visible and the flow velocity maximum as it converges into the vortex tube(Figure 3 ).

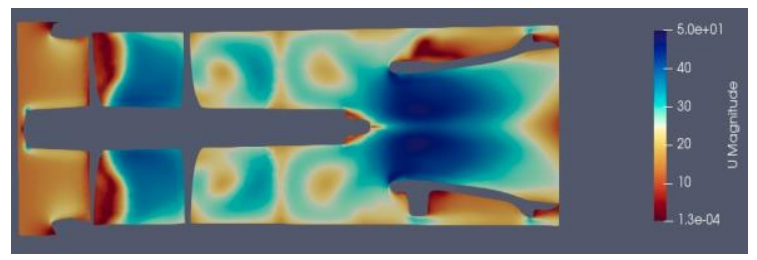

Figure 3. Velocity contour across mid-section

Figure 4 shows the streamlines across the filter. The rotating flow near the blades caused by the blades gives the particles the necessary swirl required to be pushed into the periphery. After the separation region, the majority of the particles follow the path into the annulus and gets scavenged out.

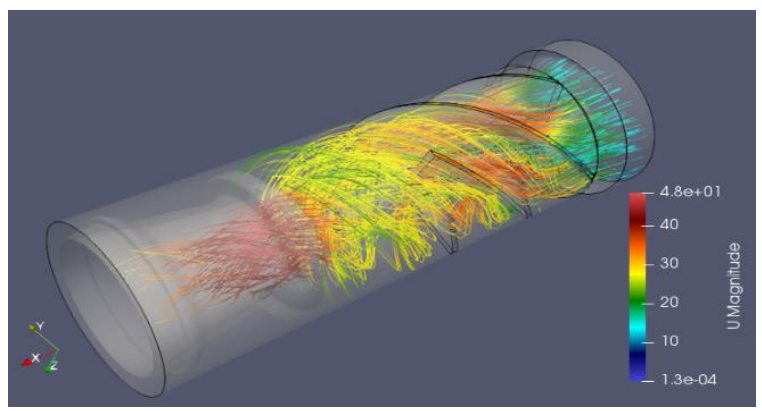

Figure 4. Streamlines across the filter

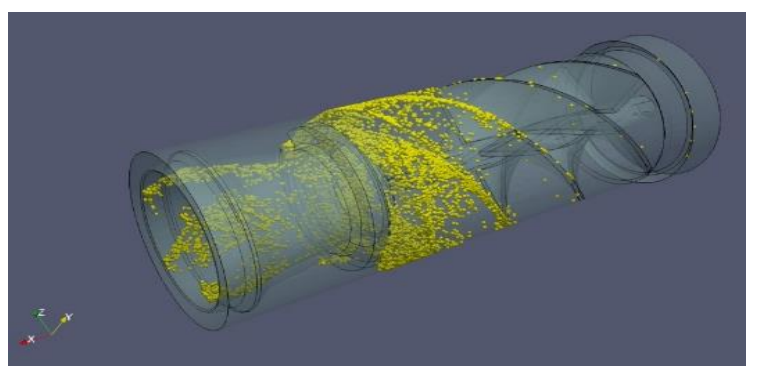

Figure 5. Particle tracks across filter for uniform inlet particle distribution

Initially a uniform particle distribution is given at the inlet and results showed that most particles are getting swirled into the periphery (Figure 5) and a smaller fraction exits through the vortex tube. But in the actual scenario, the inlet particles are having a varying diameter distribution. In order to replicate the actual condition, a distributed particle configuration given by Dziubak[9] is provided at the inlet. 


\subsection{Effect of geometric parameters}

With the objective of improving the performance of the filter, it is necessary to identify the parameters to be varied. Although flow parameters and environment conditions will have an effect on the output, those parameters are not controllable and hence kept the same for relativistic studies.

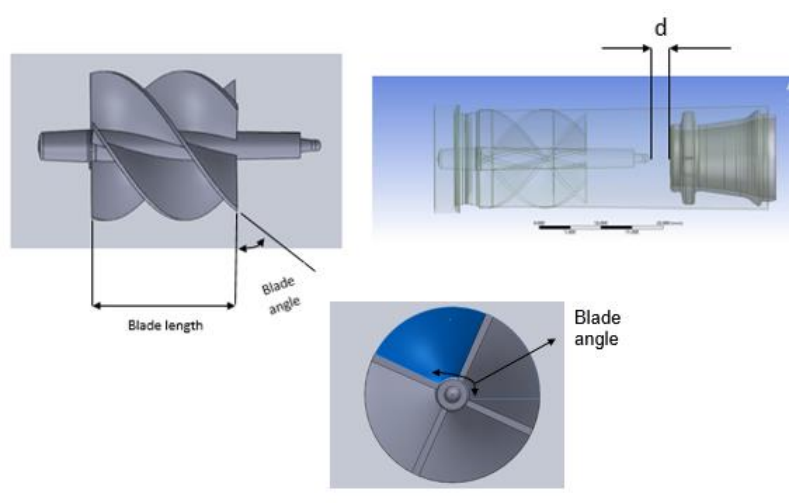

Figure 6. Geometric parameters

The axial swirl tube cyclone separator can be divided into two components, the swirl generator and the outlet tube (vortex tube). The swirl generator consists of multiple blades which are curved in the form of a helix (Figure 6).

The four major parameters which influence the cyclone performance are:

1) Number of blades

2) Blade angle

3) Blade length

4) The distance between the swirl generator and the vortex tube $(d)$

To study the individual effects of a parameter on the performance, a preliminary analysis of the variation of different parameters is done. Each parameter is varied keeping the other factors constant. The trends give a basic understanding of how the output is influenced by the geometric variation

The number of blades of the filter is taken as 3, 4 and 5 . From the plots (Figure 7(a)), we see that the pressure drop and efficiency increases with number of blades. The maximum number of blades were limited to 5 since higher pressure drops are unfavorable.

The blade angle is varied from 145 degrees to 175 degrees and the effects on the output are observed (Figure 7(b)). We observe that the pressure drop and efficiency increases with blade angle, but the variation in efficiency is smaller as compared to pressure drop.

The blade length is varied from $13 \mathrm{~mm}$ to $22 \mathrm{~mm}$. It is found to be a sensitive parameter and has a significant effect on both the pressure drop and filtration efficiency (Figure 8(a)). The pressure drop decreases with blade length, but at the cost of a decrease in filtration efficiency.
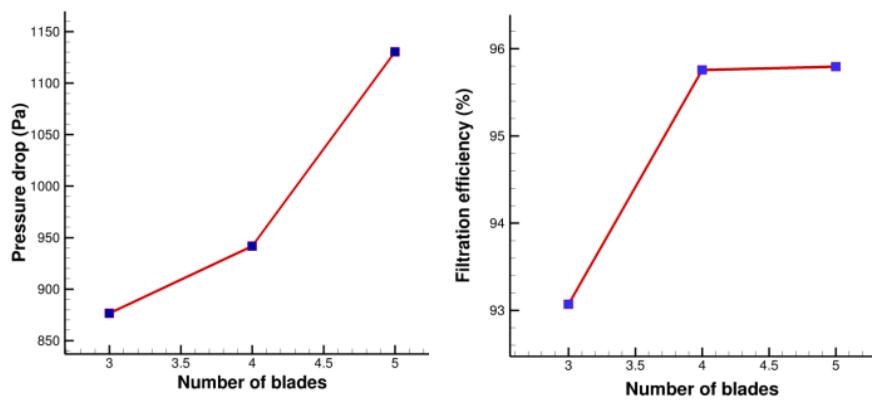

(a)
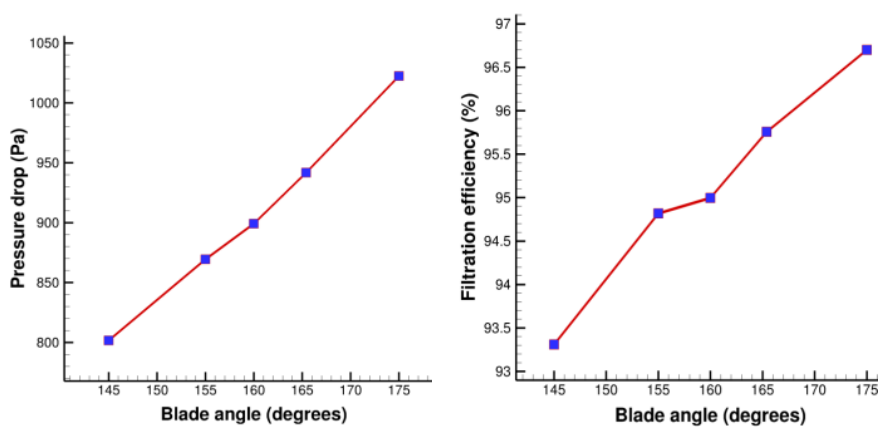

(b)

Figure 7. Variation of pressure drop and filtration efficiency with (a) Number of blades (b) Blade angle

The blade tube distance is found to have a very small effect on both the parameters (Figure $8(\mathrm{~b})$ ). Both the pressure drop and the filtration efficiency does not change significantly to have an effect on the overall performance.
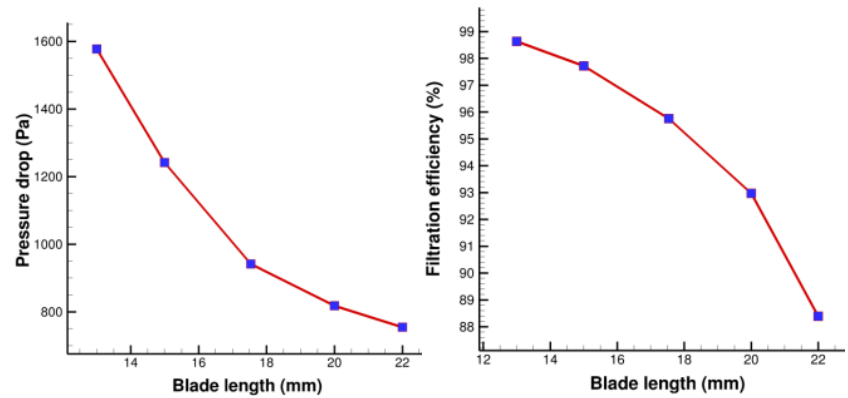

(a)
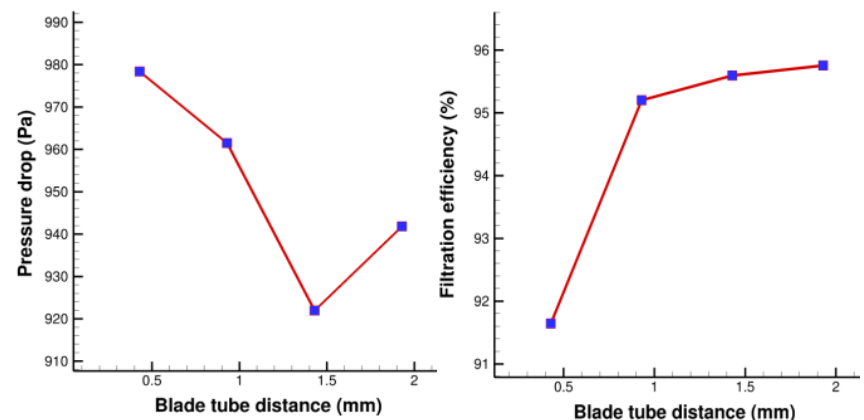

(b)

Figure 8. Variation of pressure drop and filtration efficiency with (a) Blade length (b) Blade tube distance 


\subsection{Effect of particle size on filtration efficiency}

To study about the effect of particle size on the filtration efficiency, particles of different diameters are injected with one particle diameter at a time. The heavier particles, having larger inertia gets swirled more than the lighter particles. From a varying particle distribution at the inlet, it is difficult to understand the effect of each particle size on the efficiency accurately. Hence particles of a specific sizes is injected with uniform distribution and the variation is studied.

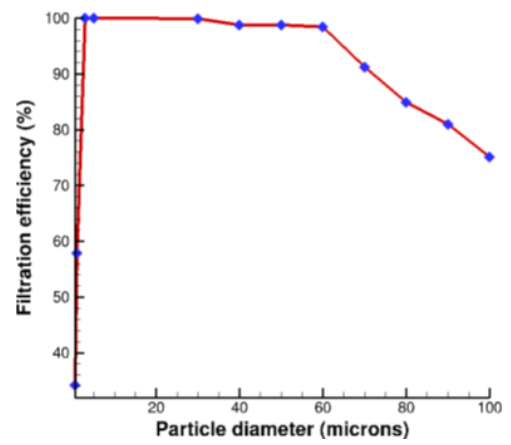

Figure 9. Variation of Filtration efficiency with particle diameter

From figure 9, we see that till a particle size of about 60 microns, the particle efficiency increases. Subsequently, there is a decrease observed in the particle efficiency with increase in particle size. To understand this anomaly, an analysis of the complete particle trajectories is to be made.

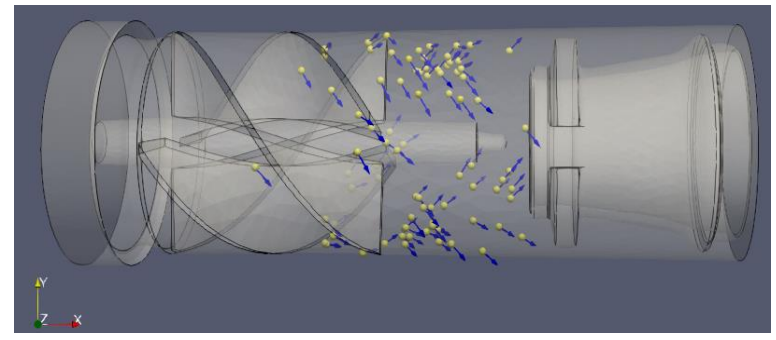

Figure 10. Particle velocity vectors at intermediate time instant for particle diameter, $\mathrm{d}=10$ microns

When the particle enters the swirl generator, it gets swirled radially outward because of the radial velocity generated due the influence of both the axial component of the flow, which carries it forward, and the radial component which causes it to swirl. If the radial velocity is very small compared to the axial velocity, by the time the particle encounters major collisions, the axial component carries it forward and hence it takes a more outward path and gets filtered off. This is the case with particles of smaller size (Figure 10).

After a particular particle size, the heavier particles having a higher radial velocity encounters more number of collisions before it comes out of the swirl generator and hence its path is heavily altered due to the collisions.

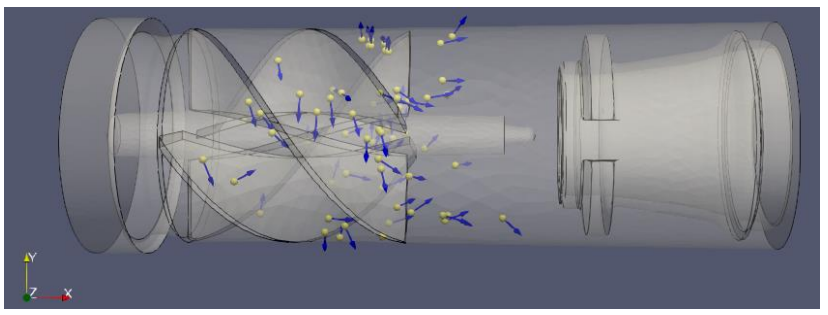

Figure 11. Particle velocity vectors at intermediate time instant for particle diameter, $\mathrm{d}=100$ microns

Also, the impact of collision on the particle is higher. Due to these effects, instead of swirling towards the periphery, a higher fraction of heavy sized particles shows a change from the original trajectory after multiple collisions and exits through the outlet tube (Figure 11) which causes a decrease in efficiency

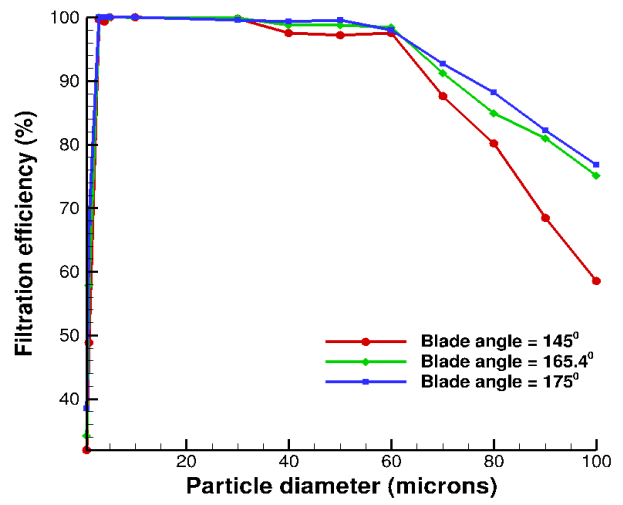

Figure 12. Filtration efficiency vs particle diameter curve with different blade angles

The size of the particle at which this effect starts to dominate depends on both the flow and geometric conditions. The main parameters affecting this cut off size are the flow velocity, filter diameter, blade length, number of blades and blade angle.

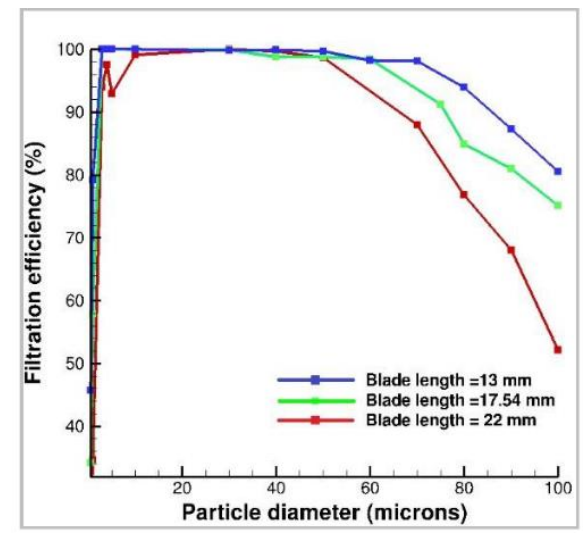

Figure 13. Filtration efficiency vs particle diameter curve with different blade lengths 
Figures 12-13 show the separation curves for different blade angle and different blade length. The radial velocity generated is proportional to the particle size. Because of the radial movement, the particle encounters collision with the filter wall and rebounds. If the number of collisions are more, the filtration efficiency may go down because the particles may deviate from the intended path (towards the periphery) and move through the outlet tube. Hence the collisions of the particles tend to play an important role in its trajectory towards the outlet.

\section{Conclusion}

An Eulerian-Lagrangian model has been used for modelling the flow through an axial cyclone separator. The turbulent flow inside the cyclone is modelled using RNG K- $\varepsilon$ closure model. A preliminary analysis of the effect of geometric parameters in the output of the filter is performed. The behaviour of the particles at different size ranges with respect to filtration efficiency were analysed in detail. The separation efficiency curves for each configuration shows that the cut off diameter range is dependent on the geometric parameters and an estimate on the diameter range can be done from the analysis. The anomalous behaviour of particles have an important implication on the filtration efficiency and hence directly affects the filter output performance.

\section{Acknowledgement}

The authors acknowledge DRDO for funding the project (No: APM1617130DRDOKARU) and CVRDE, DRDO for providing the technical specifications.

\section{References}

[1] G. Gronald. J.J. Derksen, Simulating turbulent swirling flow in a gas cyclone: A comparison of various modelling approaches, Powder Technology, 205, 160-171(2011)

[2] Weiming Peng, Alex C. Hoffmann, Separation Characteristics of Swirl-Tube Dust Separators, AIChEJournal, Vol. 50(2004)

[3] B.Wang, D.L. $\mathrm{Xu}$ and K.W.Chu, Numerical study of gas solid cyclone separator, Applied Mathematical modelling, 30:1326-1342(2006)

[4] L.Klujso, P K Songfack, M Rafaek, RK Rajamani, Design of a stationary guide vane swirl air cleaner, Minerals Engineering, vol 12, no 11,pp.1375-1392(1999)

[5] Binbin Pei, Mengting Jiang, Numerical analysis of forces exerted on particles in cyclone separators, Powder Technology, 294, 437448(2016)

[6] Sujeet Kumar Shukla, Prashant Shukla and Pradyumna Ghosh, Evaluation of Numerical Schemes for Dispersed Phase Modeling of Cyclone Separators, Engineering Applications of Computational Fluid Mechanics, 52:235-246 (2011)

[7] O. Ramachandran, P. C. Raynor and D. Leith, Collection efficiency and pressure drop for a rotary-flow cyclone, Filtration \& separation, vol. 31, pp. 631-636 (1994)

[8] Yakhot, V, Orszag, S.A, Thangam S, Gatski, T.B. \& Speziale, C.G), "Development of turbulence models for shear flows by a double expansion technique", Physics of Fluids, Vol. 4, No. 7, pp1510-1520 (1992)

[9] Tadeusz Dziubak, Performance characteristics of Air intake pleated panel filters for internal combustion engines in a two-stage configuration, Aerosol Science and Technology, 52:11, 12931307(2018)

[10] Yang Fan, Liu Ailan and Guo Xueyan, Numerical Simulation on the Performance of Axial Vane Type Gas-Liquid Separator with Different Guide Vane Structure, International Journal of Fluid Machinery and Systems, Vol 10, No 1(2017)

[11] C. Fernandes, D. Semyonov, L. L. Ferrás, J. Miguel Nóbrega, Validation of the CFD-DPM solver DPMFoam in OpenFOAM through analytical, numerical and experimental comparisons, 2018, Granular Matter, 2064(2018) 